DOI:http://dx.doi.org/10.18273/revint.v35n1-2017008

\title{
A model of competing species that exhibits zip bifurcation
}

\author{
Luis F. Echeverri ${ }^{a *}$, Óscar I. Giraldo ${ }^{b}$, EdWin Zarrazola $^{a}$ \\ ${ }^{a}$ Universidad de Antioquia, Instituto de Matemáticas, Medellín, Colombia \\ ${ }^{b}$ Universidad Nacional de Colombia, Escuela de Matemáticas, Medellín, Colombia
}

\begin{abstract}
The purpose of this paper is to present a concrete model of competing population species that exhibits a phenomenon called zip bifurcation. The Zip Bifurcation was introduced by Farkas in 1984 for a three dimensional ODE prey-predator system describing a chemostat. We will study a three dimensional system of ordinary differential equations that model the competition of two predators species for one single prey species. The system is based on concrete trigonometric functions modeling the growth rate of the prey and the functional response of the predator. The model exhibits different kinds of behavior and shows examples of the so called "competitive exclusion principle," and the competition of one "r-strategist" and one "K-strategist." Additionally, in order to illustrate the zip bifurcation, we will present some numerical simulations for our model.
\end{abstract}

Keywords: Predator prey model, Zip bifurcation, r-strategist, K-strategist MSC2010: 92D25, 92D40, 34C23, 34D20, 34A34.

\section{Un modelo de especies en competencia que exhibe bifurcación zip}

Resumen. El objetivo de este trabajo es presentar un modelo concreto de poblaciones de especies en competición que exhibe la bifurcación Zip. La bifurcación zip fue introducida por Farkas en 1984 para un sistema tridimensional de ecuaciones diferenciales ordinarias que describe un quimiostato. Estudiaremos un sistema tridimensional de ecuaciones diferenciales ordinarias que modela la competición de dos poblaciones distintas de predadores por una única población presa. El sistema usa funciones trigonométricas concretas para representar la tasa de crecimiento de la presa y la respuesta funcional del predador. El modelo exhibe diferentes clases de comportamientos y muestra ejemplos de los llamados principio de exclusión competitiva y la competición

\footnotetext{
*E-mail: lfecheve@mat.ucm.es

Received: 20 June 2016, Accepted: 22 June 2017.

Para citar este artículo: L.F. Echeverri, Ó.I. Giraldo, E. Zarrazola, A model of competing species that exhibits zip bifurcation, Rev. Integr. Temas Mat. 35 (2017), No. 1, 127-141.
} 
de un $r$-estratega contra un $k$-estratega. Adicionalmente, para ilustrar la bifurcacion zip, presentaremos algunas simulaciones numéricas.

Palabras clave: Modelo depredador-presa, Bifurcación Zip, r-estratega, Kestratega

\section{Introduction}

In this article, we introduce a tri-dimensional ordinary differential equation system for two predator population species competing against one single prey species which exhibits a bifurcation known as zip.

The phenomenon of zip bifurcation was introduced by Farkas [7] in 1984 joint with a ODE system describing the competition of two predator species for a single prey. This phenomenon is characterized by the fact that under certain conditions over the parameters the system has an one-dimensional continuum of equilibria. At low values of carrying capacity $K$, this line of equilibria is an attractor for the system and represents stable coexistence among the species; nevertheless, as $K$ is increased, the equilibria are continuously destabilized, one of the predators loses competition and only one survives with the prey.

In order to get the one dimensional continuum of equilibria and, therefore, the zip bifurcation, it is necessary to suppose that boundary parameters (boundary prey quantity) of predator species are equal for both predators. This supposition results in a structurally unstable system and, consequently, less useful to describe real particular ecosystems [18]. Nevertheless, this supposition makes it possible to identify one of the predators as a $K$-strategist and the other as a $r$-strategist. Intuitively, an $r$-strategist is a species that tries to survive throughout a high birth rate and a $K$-strategist is a species that consumes little food, has low birth rate, and can maintain its siblings with little food supply.

It is important to note that the models introduced by Farkas have been originated in a micro-organism test tube named chemostat [3], [4], [5], [20], [21]. In such a tube, food (prey species) is given to the two predator species at a constant rate and the output quantity of the system has to be equal to the input one; this output is made of non consumed food and waste products. Inside the system, cells are produced. In the earlier mathematical models for chemostat, food given to predator species was a prey species that was modeled according to a logistic differential equation in absence of predators [20]. Most of such models could exhibit certain kind of behavior, which has been defined as the competitive exclusion principle. According to this principle, two predators having a single common prey cannot survive and the "less fit" dies out.

Farkas has proven (see [8], [9], [10]) that the phenomenon called zip bifurcation is general, in the sense that it is present in all tri-dimensional models that satisfy certain theoretical conditions. From Farkas' research, the zip bifurcation phenomenon has been studied from several points of view: particularization of Farkas' general system for models including numerical experiments [6]; 3-dimensional competition models with generalized Holling type III functional response for the predators [19]; generalization to four-dimensional systems coming from economy and politology [2] and from population dynamics [11]; generalization to $n$-dimensional ordinary differential systems with and without diffusion

[Revista Integración, temas de matemáticas 
[12], [13], [14]; tri-dimensional predator-prey model with a delay term for the growth of the prey [15]; and non-smooth systems that exhibit a corresponding non-smooth zip bifurcation [1].

It is important to remark that the most usual functions to build equations that model competing populations species exhibiting zip bifurcation are of two kinds (see [1], [2], [6][15], [19]): The logistic function: $G(S)=\gamma S\left(1-\frac{S}{K}\right)$ for the per-capita growth rate of the prey, where $S$ is the prey density and $\gamma>0$ denotes the maximum growth rate, assuming that the carrying capacity of the habitat $K>0, K \geq S \geq 0$ and the Holling-family functions: $P(S)=m \frac{S^{n}}{a^{n}+S^{n}}$ for the functional response of the predators, where $n \geq 1$ is an arbitrary integer that defines the type of Holling function, $m>0$ is the supremum of $P$, and $a>0$ is the half-saturation constant. The logistic function describes the situation when the increasing rate is slow at the beginning, accelerates in the middle and slows up at the end. Holling-family functions are increasing, bounded and depending on the integer $n \geq 1$; they could describe quick/slow increase $(n=1 / n>1)$ at low values of the variable and always slows down at higher values.

In this paper, we consider a concrete model of two predator species competing for a single prey with different per-capita growth rate for the prey and functional response of the predators. Those are $G(S)=\gamma S\left(1-\frac{3}{\pi} \operatorname{arcsec}\left(\frac{S}{K}+1\right)\right)$ for the per capita growth rate of the prey, and $P(S)=\hat{m} \cdot \operatorname{arcsec}\left(1+\frac{S}{\alpha_{i}}\right)$ for the functional response of the predators $(i=$ $1,2)$. This per-capita growth rate of the prey, in contrast with the logistic function, has the property of reaching its maximum before, as the variable increases, and this maximum is lower than the one of the logistic function; nevertheless, the remaining properties are the same as the logistic function. The functional response of the predator is strictly increasing, bounded, and concave down, and in the same way as Holling family functions slows down at higher values of the variable. But in contrast with those functions, the functional response of the predators describes a quicker increase at low values. Finally, the supremum of our $P$ is greater than the Holling function one, and this supremum is not equal to our $\hat{m}$, which is defined by: $\hat{m}=\frac{A}{B \alpha_{i}+C}$.

We will begin introducing the general conditions mentioned by Farkas in [8] for the zip bifurcation (Section 2); then we will introduce the concrete model and show that this one meets such conditions, and we will make a comment about the intuitive meaning of each one (Section 3). Moreover, with this model, we will carry out qualitative analysis as well as numerical experiments (Section 4). Numerical experiments play a very important role in the sense that few publications on zip bifurcation show how the zip behaves, given particular values of the bifurcation parameter and initial conditions for the species. We will present numerical experiments for the zip bifurcation as well as for Hopf bifurcation (Section 5), which occur when we consider the system restricted to the prey and only one predator species, living in the habitat, with precise values of the carrying capacity $K$.

\section{Zip-bifurcation conditions}

The dynamics of an ecosystem made of two predator species for a single prey species, that eventually shows a zip bifurcation, can be described by the following system of differential equations (see [8]):

Vol. 35, No. 1, 2017] 


$$
\begin{aligned}
& \dot{S}=\gamma S g(S, K)-x_{1} p\left(S, \alpha_{1}\right)-x_{2} p\left(S, \alpha_{2}\right), \\
& \dot{x}_{1}=x_{1} p\left(S, \alpha_{1}\right)-d_{1} x_{1}, \\
& \dot{x}_{2}=x_{2} p\left(S, \alpha_{2}\right)-d_{2} x_{2} .
\end{aligned}
$$

We denote the quantity of prey species in time by $S(t)$ and the respective quantities of predators by $x_{1}(t)$ and $x_{2}(t)$. In the same way, we denote by $\gamma$ the intrinsic growth rate of the prey; by $K$ the carrying capacity of the habitat; by $m_{i}$ and $d_{i}$, (with $i=1,2$ ) the maximum birth and death rate of $i$-th predator, respectively. The per-capita growth rate of the prey in absence of predators is $\gamma g(S, K)$. The per-capita growth rate of the $i^{t h}$ predator is $p\left(S, \alpha_{i}\right)$ for $i=1,2$. We note that predators' death rate is assumed to be constant and all the parameters defined in the system (1) are positive. Finally, we denote by $\alpha_{i}>0$ (with $i=1,2$ ) the so called half saturation constant of the respective predator. The interpretation for this parameter is: when the prey species reaches the $\alpha_{i}$-value the per capita birth rate of the $i^{\text {th }}$ predator, species reaches a half of the maximum birth rate $m_{i}$.

The functions $g$ and $p$ must satisfy the following conditions (according to Butler and Farkas):

$$
\begin{aligned}
& \text { (I) } \quad g \in C^{2}((0, \infty) \times(0, \infty), \mathbb{R}), \quad g \in C^{0}([0, \infty) \times(0, \infty), \mathbb{R}), \\
& \quad p \in C^{1}((0, \infty) \times(0, \infty), \mathbb{R}) \text { and } p \in C^{0}([0, \infty) \times(0, \infty), \mathbb{R}) . \\
& \text { (II) } g(0, K)=1 ; g_{S}^{\prime}(S, K)<0<g_{S K}^{\prime \prime} \quad \text { for } \quad S \geq 0, \quad K>0 . \\
& \text { (III) } \quad \lim _{K \rightarrow \infty} g_{S}^{\prime}(S, K)=0 . \\
& \text { (IV) } \quad(K-S) g(S, K)>0 \text { for } S \geq 0, \quad K>0, \quad S \neq K . \\
& \text { (V) } p(0, \alpha)=0, p_{S}^{\prime}(S, \alpha)>0 \text { for } S>0, \quad \alpha>0 . \\
& \text { (VI) } p_{S}^{\prime}(S, \alpha)<\frac{p(S, \alpha)}{S} \text { for } S>0, \alpha>0 . \\
& \text { (VII) } p_{\alpha}^{\prime}(S, \alpha)<0 \text { for } S>0, \quad \alpha>0 .
\end{aligned}
$$

We additionally suppose that

$$
\alpha_{2}<\alpha_{1}
$$

From condition (2) and by (VII), we have that

$$
p\left(S, \alpha_{1}\right)<p\left(S, \alpha_{2}\right) \text { for all } S>0 ;
$$

furthermore, we assume that

$$
d_{1}<d_{2}
$$

and the following two conditions:

$$
\begin{gathered}
p\left(\lambda, \alpha_{i}\right)=d_{i} \text { for } i=1,2, \\
K>\lambda
\end{gathered}
$$

[Revista Integración, temas de matemáticas 
where $\lambda$ is the prey threshold quantity for the predator species. The system (1) satisfying conditions (I) - (VII) and (2) - (5) represents a whole class of models of competing species that exhibit zip bifurcation (see [8]). These models are classified in three subclasses that are called natural, artificial, and degenerated when the partial derivative

$$
\frac{\partial}{\partial S}\left[\frac{p\left(S, \alpha_{2}\right)}{p\left(S, \alpha_{1}\right)}\right]_{S=\lambda}
$$

is lower, greater or equal to zero, respectively. We want to show that our concrete model is natural. This means that the advantage of species 2 over species 1 as expressed by $p\left(S, \alpha_{1}\right)<p\left(S, \alpha_{2}\right)$, for all $S>0$, decreases as the quantity of the prey increases.

\section{The concrete model}

We propose a concrete model of two predators species competing for a single prey species. In this model, the growth rate of the prey and the functional response of the predators are assumed to be of trigonometric type. The model is represented by the following system of differential equations:

$$
\begin{aligned}
\dot{S}= & \gamma S\left(1-\frac{3}{\pi} \operatorname{arcsec}\left(\frac{S}{K}+1\right)\right)-x_{1} \frac{A}{B \alpha_{1}+C} \operatorname{arcsec}\left(1+\frac{S}{\alpha_{1}}\right) \\
& -x_{2} \frac{A}{B \alpha_{2}+C} \operatorname{arcsec}\left(1+\frac{S}{\alpha_{2}}\right), \\
\dot{x_{1}=} & x_{1} \frac{A}{B \alpha_{1}+C} \operatorname{arcsec}\left(1+\frac{S}{\alpha_{1}}\right)-d_{1} x_{1}, \\
\dot{x_{2}=} & x_{2} \frac{A}{B \alpha_{2}+C} \operatorname{arcsec}\left(1+\frac{S}{\alpha_{2}}\right)-d_{2} x_{2} .
\end{aligned}
$$

In this system $A>0, B \geq 0, C \in \mathbb{R}$. We will prove, step by step, that this system satisfies the Butler-Farkas conditions (I)-(VII) and we will assume conditions (2)-(5). In fact, from this system, we notice that the per capita growth rate of prey species is: $g(S, K)=$ $\left(1-\frac{3}{\pi} \operatorname{arcsec}\left(\frac{S}{K}+1\right)\right)$, with $S \geq 0$ and $K>0$; it is clear that $g(K, K)=0$. This means that when the number of prey species reaches the carrying capacity, the prey growth is lower than or equal to zero. Let's check condition (II): $g(0, K)=1-\frac{3}{\pi} \operatorname{arcsec}(1)=1$; this means that the maximum specific growth rate of the prey is reached when $S=0$, $x_{1}=0$ and $x_{2}=0$ and this maximum rate is equal to $\gamma>0$.

Furthermore, since $\left(\frac{S}{K}+1\right)>1$, we have $g_{S}^{\prime}(S, K)=-3\left(\pi K\left(\frac{S}{K}+1\right) \sqrt{\left(\frac{S}{K}+1\right)^{2}-1}\right)^{-1}<0$. Moreover,

$$
g_{S K}^{\prime \prime}(S, K)=\frac{3 S}{\pi}\left(\frac{K^{2}-K S-S^{2}}{(S+K)^{2}\left(S^{2}+2 S K\right)^{\frac{3}{2}}}\right)>0 ;
$$

since $g_{S}^{\prime}(S, K)<0$, and given that $\lim _{K \rightarrow \infty} g_{S}^{\prime}(S, K)=\frac{1}{\pi} \lim _{K \rightarrow \infty} \frac{-K}{S+K} \lim _{K \rightarrow \infty} \frac{3}{\sqrt{S^{2}+2 S K}}=0$, then $g_{S}^{\prime}(S, K)$ must be a increasing function of $K$; so, $g_{S K}^{\prime \prime}(S, K)>0$. Besides, the factor $\left(K^{2}-K S-S^{2}\right)$ is positive, since if we have a fixed $S$ and we make $K$ bigger, then $K^{2}$ dominates over $-\left(K S+S^{2}\right)$.

Vol. 35, No. 1, 2017] 
The condition (III) means that high $K$ variations in the prey quantity have a non significant effect over the growth rate.

Let's check condition (IV): We have two possibilities: $K>S$ and $K<S$.

1. If $K>S$, then: $\frac{S}{K}<1$, which implies $\frac{S}{K}+1<2$; given that the function $\operatorname{arcsec}$ is an increasing one, $\operatorname{arcsec}\left(\frac{S}{K}+1\right)<\operatorname{arcsec}(2)$, hence $1-\frac{3}{\pi} \operatorname{arcsec}\left(\frac{S}{K}+1\right)>0$, and $(K-S)\left(1-\frac{3}{\pi} \operatorname{arcsec}\left(\frac{S}{K}+1\right)\right)>0$.

2. If $K<S$, then $\frac{S}{K}>1$, which implies $\frac{S}{K}+1>2 ;$ therefore, $\operatorname{arcsec}\left(\frac{S}{K}+1\right)>\operatorname{arcsec}(2)$, and $1-\frac{3}{\pi} \operatorname{arcsec}\left(\frac{S}{K}+1\right)<0$; hence, we have $(K-S)\left(1-\frac{3}{\pi} \operatorname{arcsec}\left(\frac{S}{K}+1\right)\right)>0$; this means that if there are not predators and if $S$ is lower than the carrying capacity, $K$, then the growth rate of the prey species is positive, and if $S$ is higher than $K$, then the growth rate of the prey species is negative.

We return now to system (1). Note that the per-capita growth rate of the predator is

$$
p(S, \alpha)=\frac{A}{B \alpha+C} \operatorname{arcsec}\left(1+\frac{S}{\alpha}\right), \text { with } A>0, B \geq 0, C \in \mathbb{R} .
$$

Now, let's check condition $(\mathrm{v}): p(0, \alpha)=\frac{A}{B \alpha+C} \operatorname{arcsec}(1)=0$; this means that if there is no a prey, predators growth rate is zero. Furthermore, if $C>0$, we have that $p_{S}^{\prime}(S, \alpha)=$ $A \times\left(\alpha(B \alpha+C)\left(1+\frac{S}{\alpha}\right) \sqrt{\left(1+\frac{S}{\alpha}\right)^{2}-1}\right)^{-1}>0$; this means that the per capita birth rate of predators is an increasing function of the prey quantity.

Now let's check condition (VI): this condition is true if

$$
\frac{A}{B \alpha+C} \frac{1}{\left(1+\frac{S}{\alpha}\right) \sqrt{\left(1+\frac{S}{\alpha}\right)^{2}-1}} \frac{1}{\alpha}<\frac{A}{B \alpha+C} \frac{\operatorname{arcsec}\left(1+\frac{S}{\alpha}\right)}{S},
$$

which is equivalent to

$$
\alpha \sqrt{\frac{S}{2 \alpha+S}}<(\alpha+S) \operatorname{arcsec}\left(1+\frac{S}{\alpha}\right)
$$

taking $f_{\alpha}(S)=(\alpha+S) \operatorname{arcsec}\left(1+\frac{S}{\alpha}\right)-\alpha \sqrt{\frac{S}{2 \alpha+S}}$, obviously $f_{\alpha}(0)=0$. Proving that this function is an increasing one, condition (VI) will be true:

$$
f_{\alpha S}^{\prime}(S)=\operatorname{arcsec}\left(1+\frac{S}{\alpha}\right)+\frac{\alpha(\alpha+S)}{\sqrt{2 S \alpha+S^{2}}(2 \alpha+S)}>0
$$

this condition means that if $p$ is a strictly concave function of $S$ (for every $\alpha>0$ ) the last inequality holds, with the only possible exception of isolated points in which the equality holds.

Let's check condition (VII):

$$
p_{\alpha}^{\prime}(S, \alpha)=\frac{-B A}{(B \alpha+C)^{2}} \operatorname{arcsec}\left(1+\frac{S}{\alpha}\right)<0, \quad \text { since } A>0 \text { and } B \geq 0
$$

[Revista Integración, temas de matemáticas 
this condition means that the predators birth rate is a decreasing function of $\alpha$, and so the greater the value of $\alpha$, the greater the food quantity is needed to keep the same birth rate of a specific predator. If $p$ is bounded for a fixed $\alpha>0$, then we define the maximum birth rate of predator as $m_{i}:=\sup _{S>0} p\left(S, \alpha_{i}\right)$; hence,

$$
m_{i}=\lim _{S \rightarrow \infty} p\left(S, \alpha_{i}\right)
$$

so, the meaning of mean saturation constant $\alpha_{i}$ is that the value of the per-capita birth rate of the $i^{\text {th }}$ predator is $\frac{2}{3} m_{i}$ when $S=\alpha_{i}$. This is so because

$$
m_{i}=\lim _{S \rightarrow \infty} p\left(S, \alpha_{i}\right)=\frac{A}{B \alpha_{i}+C} \frac{\pi}{2}
$$

thus $p\left(\alpha_{i}, \alpha_{i}\right)=\frac{2}{3} m_{i}$; in our particular case: $\lim _{S \rightarrow \infty} p\left(S, \alpha_{i}\right)=\frac{A}{B \alpha_{i}+C} \frac{\pi}{2}$.

From now on, we will only study the case $\alpha_{1}>\alpha_{2}>0$, and ignore the case $\alpha_{1}=\alpha_{2}$ because it is less interesting. So, we suppose that the Butler-Farkas condition (2) holds for system (6). From the previous observation, it is clear that $p\left(S, \alpha_{1}\right)=$ $\frac{A}{B \alpha_{1}+C} \operatorname{arcsec}\left(1+\frac{S}{\alpha_{1}}\right)<\frac{A}{B \alpha_{2}+C} \operatorname{arcsec}\left(1+\frac{S}{\alpha_{2}}\right)=p\left(S, \alpha_{2}\right)$. This condition means that regardless the prey quantity, predator 2 birth rate is greater than predator 1 birth rate. In other words, in order to reach the same birth rate, predator 1 needs more prey quantity than predator 2.

Now we shall assume that condition (3) holds, that is, $d_{1}<d_{2}$. Otherwise, if $d_{1} \geq d_{2}$, $p\left(S, \alpha_{1}\right)-d_{1}<p\left(S, \alpha_{2}\right)-d_{2}$, then predator 2 birth rate would be greater than predator 1 birth rate; in this case we can prove that predator 2 beats predator 1 in the competition for food, and this population species will die out. Clearly we do not want this to happen.

One important feature of each predator species is the so called threshold prey quantity, denoted by $\lambda_{i}(i=1,2)$. This parameter indicates that when $S=\lambda_{i}$, then $x_{i}^{\prime}=0$, and so, if $S>\lambda_{i}$ then the $i^{\text {th }}$ predator birth rate is positive. Clearly, the lower $\lambda_{i}$, the better survival for $i^{\text {th }}$ predator.

In this paper, we shall assume $\lambda_{1}=\lambda_{2}$, that is, both predator species have the same threshold prey quantity, regardless that each one of them reaches this number by different means.

\section{Main results}

In order to work with the threshold prey quantity $\lambda$, we can see $\lambda$ as a function of $\alpha_{i}, d_{i}$ in a particular domain; for instance, in the unit square $[0,1] \times[0,1]$, and so for a given $\lambda$, we can find infinitely many pairs $\left(\alpha_{i}, d_{i}\right)$ such that $f\left(\alpha_{1}, d_{1}\right)=f\left(\alpha_{2}, d_{2}\right)=\lambda$.

Clearly, from the per-capita growth rate of the predators, we have

$$
f\left(\alpha_{i}, d_{i}\right)=\alpha_{i}\left(\sec \left(\frac{d_{i}\left(B \alpha_{i}+C\right)}{A}\right)-1\right) .
$$

For sake of simplicity, we will choose $A=2, B=C=1$, and $\left(\alpha_{i}, d_{i}\right)$ in the unit square. From this, we have a continuous function of $\alpha$ and $d$ (see Figure 1), and by continuity,

Vol. 35, No. 1, 2017] 

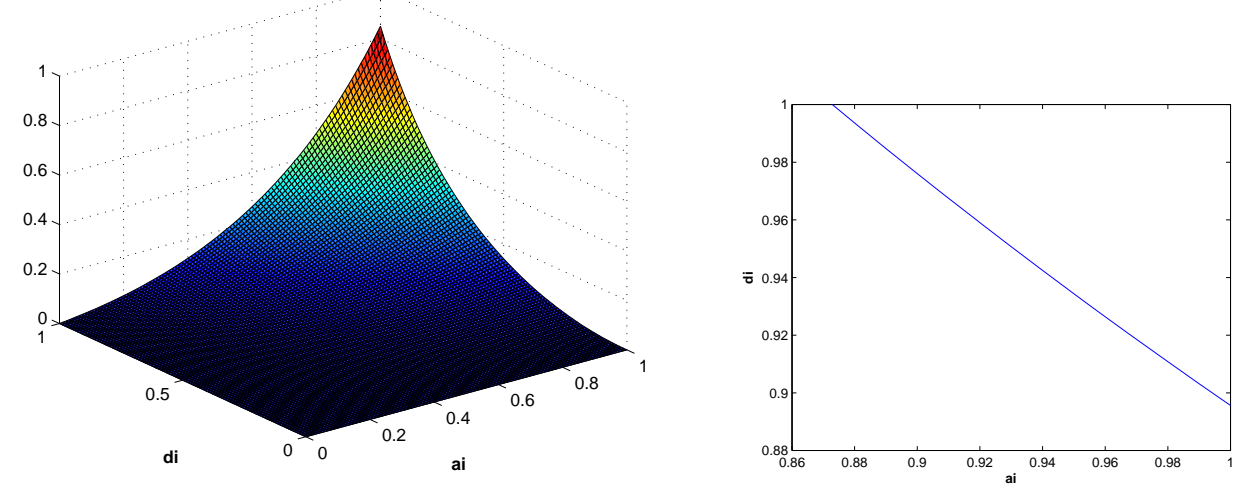

Figure 1. Threshold prey quantity $\lambda$ as function of $\alpha_{i}$ and $d_{i}$ in the unit square, and the intersection of the surface with an horizontal plane at $\lambda=0.6$.

for a given $\lambda$ between min and max of $f$, there exists at least one pair $\left(\alpha_{i}, d_{j}\right)$ such that $f\left(\alpha_{i}, d_{j}\right)=\lambda$.

According to conditions (2) and (3), for a zip bifurcation to occur, we choose $\alpha_{2}<\alpha_{1}$ and $d_{1}<d_{2}$. We will consider $\lambda=0.6$, and for this value, there exists a curve which represents the infinitely many points that satisfy condition (4), i.e., $p\left(\lambda, \alpha_{i}\right)=d_{i}$ for $i=1,2$. So we have infinitely many possibilities to choose $\alpha_{2}<\alpha_{1}$ and $d_{1}<d_{2}$, as shown in the graph of the mentioned curve (see Figure 1).

For instance, we can take the curve extreme values, that is the points $\left(1, d_{i}\right)$ and $\left(\alpha_{i}, 1\right)$, $i=1,2$. Now, according to the numerics calculations, those values are $(1,0.8957)$ and $(0.8729,1)$. The reader can easily verify that for those values, conditions $(2),(3)$, and (4) hold.

We mentioned that our model was natural; that is right. It is possible to prove it; nevertheless, it is time-consuming to prove that if $\alpha_{1}>\alpha_{2}>0$ and $S>0$, then $\frac{\partial}{\partial S}\left(\frac{p\left(S, \alpha_{2}\right)}{p\left(S, \alpha_{1}\right)}\right)<0$ for all $S>0$. We can conclude that our model is natural, which means that the ratio between birth rates $(>1)$ is decreasing, in particular in a neighborhood of $S=\lambda$. As a consequence, the advantage of predator species 2 over predator species 1 $\left(p\left(S, \alpha_{1}\right)<p\left(S, \alpha_{2}\right)\right.$ for all $\left.S>0\right)$ decreases when the prey quantity increases.

From all we have said, we can conclude that (6) is a natural system in which ButlerFarkas conditions (I)-(VII) holds. Conditions (2)-(5) are assumed, then the system must exhibit a zip bifurcation, just as the following theorem (see [8] for the general case) shows in the particular case.

Theorem 4.1. Given that Butler-Farkas conditions (I)-(VII) holds for system (6) and also that it is a natural system, then there exists $K_{1}>0$ and $K_{2}>0$, with $\lambda<K_{1}<K_{2}<\infty$, such that for $K \in\left(\lambda, K_{1}\right)$ every point in the segment

$$
\begin{array}{r}
L_{K}=\left\{\left(S, x_{1}, x_{2}\right): S=\lambda, x_{1} \geq 0, x_{2} \geq 0, \frac{A x_{1}}{B \alpha_{1}+C} \operatorname{arcsec}\left(1+\frac{S}{\alpha_{1}}\right)+\right. \\
\left.\frac{A x_{2}}{B \alpha_{2}+C} \operatorname{arcsec}\left(1+\frac{S}{\alpha_{2}}\right)=\gamma \lambda\left(1-\frac{3}{\pi} \operatorname{arcsec}\left(1+\frac{\lambda}{K}\right)\right)\right\}
\end{array}
$$

[Revista Integración, temas de matemáticas 
is stable (in the Liapunov sense). For $K \in\left(K_{2}, \infty\right)$, system (6) has no stable equilibria in the space $\left\{\left(S, x_{1}, x_{2}\right): S \geq 0, x_{1} \geq 0, x_{2} \geq 0\right\}$. For $K \in\left(K_{1}, K_{2}\right)$, the point $\left(\lambda, x_{1}(K), x_{2}(K)\right)$ divides $L_{K}$ in two parts (one of which could be empty): points of $L_{K}$ to the left of this point are unstable; points of $L_{K}$ to the right of this point are stable in the Liapunov sense.

Proof. We follow the ideas as in [8]. Given that the system (6) is a particular case of system (1), we can rewrite $L_{K}$ as follows:

$$
L_{K}=\left\{\left(S, x_{1}, x_{2}\right): p\left(\lambda, a_{1}\right) x_{1}+p\left(\lambda, a_{2}\right) x_{2}=\gamma \lambda g(\lambda, K), S=\lambda, x_{1} \geq 0, x_{2} \geq 0\right\} .
$$

Linearizing the system (1) at any point $\left(\lambda, \xi_{1}, \xi_{2}\right) \in L_{K}$, we have the characteristic polynomial of the linearized system:

$$
\begin{aligned}
D(\mu)=\mu\left[\mu^{2}+\left\{\xi_{1} p_{s}^{\prime}\left(\lambda, a_{1}\right)+\xi_{2} p_{s}^{\prime}\left(\lambda, a_{2}\right)\right.\right. & \left.-\gamma g(\lambda, K)-\gamma \lambda g_{s}^{\prime}(\lambda, K)\right\} \mu \\
& \left.+\xi_{1} p\left(\lambda, a_{1}\right) p_{s}^{\prime}\left(\lambda, a_{1}\right)+\xi_{2} p\left(\lambda, a_{2}\right) p_{s}^{\prime}\left(\lambda, a_{2}\right)\right] .
\end{aligned}
$$

Clearly $\mu=0$ is an eigenvalue. The polynomial between in square brackets is stable, if the remaining two eigenvalues have negative real part, that is, if and only if $\xi_{1} p_{s}^{\prime}\left(\lambda, a_{1}\right)+\xi_{2} p_{s}^{\prime}\left(\lambda, a_{2}\right)>\gamma\left[g(\lambda, K)+\lambda g_{s}^{\prime}(\lambda, K)\right]$. We can rewrite the last expresion as: $\gamma \lambda g(\lambda, K)+\gamma \lambda^{2} g_{s}^{\prime}(\lambda, K)<\left[\lambda p_{s}^{\prime}\left(\lambda, a_{1}\right)-p\left(\lambda, a_{1}\right)\right] \xi_{1}+\left[\lambda p_{s}^{\prime}\left(\lambda, a_{2}\right)-p\left(\lambda, a_{2}\right)\right] \xi_{2}+$ $p\left(\lambda, a_{1}\right) \xi_{1}+p\left(\lambda, a_{2}\right) \xi_{2}$.

Given that $\left(\xi_{1}, \xi_{2}\right)$ belongs to $L_{K}$, we get:

$$
\left[p\left(\lambda, a_{1}\right)-\lambda p_{s}^{\prime}\left(\lambda, a_{1}\right)\right] \xi_{1}+\left[p\left(\lambda, a_{2}\right)-\lambda p_{s}^{\prime}\left(\lambda, a_{2}\right)\right] \xi_{2}<-\gamma \lambda^{2} g_{s}^{\prime}(\lambda, K) .
$$

By condition (VI), the left side of (8) is positive for all $\left(\lambda, \xi_{1}, \xi_{2}\right) \in L_{K}$. In view of (II) and (III), the right side is positive, decreases, and tends to zero when $K \rightarrow \infty$.

Define the line segment

$$
\begin{aligned}
B_{K}=\left\{\left(S, x_{1}, x_{2}\right):\left[\lambda p_{s}^{\prime}\left(\lambda, a_{1}\right)\right.\right. & \left.-p\left(\lambda, a_{1}\right)\right] x_{1}+\left[\lambda p_{s}^{\prime}\left(\lambda, a_{2}\right)-p\left(\lambda, a_{2}\right)\right] x_{2} \\
& \left.=-\gamma \lambda^{2} g_{s}^{\prime}(\lambda, K), S=\lambda, x_{1} \geq 0, x_{2} \geq 0\right\} .
\end{aligned}
$$

Now, we determine the point of intersection of lines $L_{K}$ and $B_{K}$. Denoting this point by $\left(x_{1}(K), x_{2}(K)\right)$, we obtain (for $\left.i=1,2\right)$ :

$$
x_{i}(K)=(-1)^{i} \gamma \frac{-\lambda g_{s}^{\prime}(\lambda, K) p\left(\lambda, a_{3-i}\right)+g(\lambda, K)\left[\lambda p_{s}^{\prime}\left(\lambda, a_{3-i}\right)-p\left(\lambda, a_{3-i}\right)\right]}{p\left(\lambda, a_{2}\right) p_{s}^{\prime}\left(\lambda, a_{1}\right)-p_{s}^{\prime}\left(\lambda, a_{2}\right) p\left(\lambda, a_{1}\right)} .
$$

Provided our particular model is natural, the denominator is positive. Moreover, the conditions imposed upon the functions $g$ and $p$ imply $x_{1}(\lambda)<0, x_{2}(\lambda)>0, \lim _{K \rightarrow \infty} x_{1}(K)>0$ and $\lim _{K \rightarrow \infty} x_{2}(K)<0$. From this, we can conclude that $x_{1}(\cdot)$ is a monotonic increasing function of $K$ and that $x_{2}(\cdot)$ is a monotonic decreasing function of $K$, so, by continuity,

Vol. 35, No. 1, 2017] 

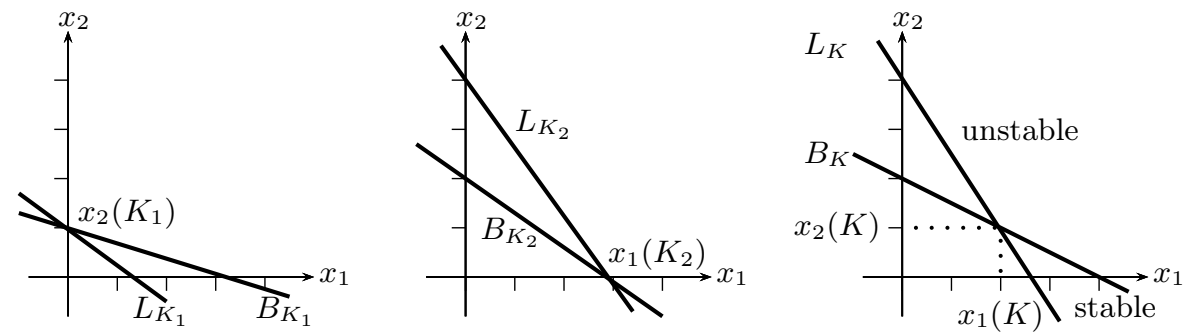

Figure 2. Points of intersection between the lines $B_{K}$ and $L_{K}$ at extreme values of $K$ and at an intermediate value.

if we increase $K$, there exists a "first" $K_{1}>\lambda$ at which $x_{1}\left(K_{1}\right)=0$. Analogously, if we continue increasing $K$, there exists a "last" $K_{2}>K_{1}$ at which $x_{2}\left(K_{2}\right)=0$ (see Figure 2).

This means that if $K \in\left(\lambda, K_{1}\right)$, then $B_{k}$ and $L_{K}$ intersect outside the positive octant in such a way that $L_{K}$ is "below" $B_{K}$, and so all the points of $L_{K}$ satisfy (8); therefore, all those points are stable. In the other extreme case, if $K \in\left(K_{2}, \infty\right)$, then $B_{k}$ and $L_{K}$ intersect outside the positive octant in such a way that $L_{K}$ is above $B_{K}$ and so no points of $L_{K}$ in the positive quadrant satisfy (8); therefore, all those points are unstable.

Now, if $K \in\left(K_{1}, K_{2}\right)$ and $\left(\lambda, x_{1}(K), x_{2}(K)\right) \in L_{K}$ belong to the positive octant, then this point divides $L_{K}$ in two parts: in the left part, condition (8) does not hold and, therefore, those points are unstable; in the right part, condition (8) is valid, and, therefore, those points are stable. We can conclude that if $K$ is increased from $K_{1}$ to $K_{2}$, the point $\left(\lambda, x_{1}(K), x_{2}(K)\right)$ move steadily along $L_{K}$ from the left end to the right end, while the segment $L_{K}$ undergoes a parallel displacement "upwards". In the process, points left behind by $\left(\lambda, x_{1}(K), x_{2}(K)\right)$ become destabilized. This phenomenon is called a zip bifurcation.

\section{Computer simulations}

In Figure 3, we present numerical solutions for our model. We can see in the first case (Figure 3a) that the one dimensional continuum of equilibria is stable, and so all the populations will survive in the long term. This means that if food is limited, both predators may live together in the long run in a steady state. Thus it depends on the initial values of the population species. In the second case (Figure $3 \mathrm{~b}$ ), we see that $K$ has indefinitely grown $\left(K>K_{2}\right)$, then all the stable points of the system become unstable. This phenomenon is known as enrichment paradox, because if food is abundant and the prey quantity is also abundant, then, contrary to intuition, one of the two predator species dies out and the other predator species will remain in the prey habitat. In the last case (Figure 3c), one part of the one-dimensional continuum of equilibria is unstable and the remainder is stable. From the point of view of the competition, as the quantity of available food is increasing, the K-strategist is losing competition and those equilibria, where the relative quantity of K-strategist is high compared to the quantity of r-strategist are the first to be destabilized.

It is important to exhibit the bifurcation of the two end points of the segment $L_{K}$. For $K>\lambda$ our system has an equilibrium point in its interior of the positive quadrant of each 
coordinate plane $S, x_{i}(i=1,2)$. The next theorem agrees with the respective numerical results shown in Figure 4.

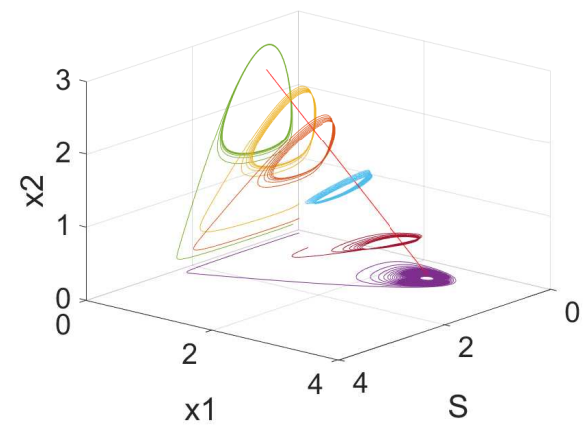

(a)

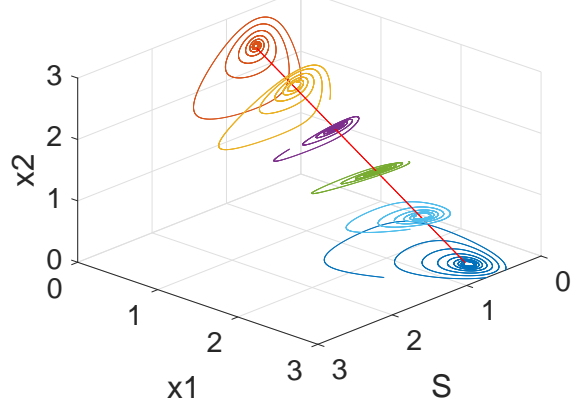

(b)

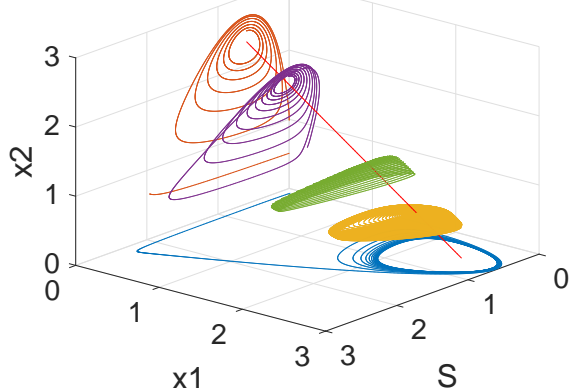

(c)

Figure 3. Numerical solutions around $L_{K}$ (in red) for $K=K_{1} ; K=K_{2}$; and $K_{1}<K<K_{2}$. Those solutions were generated using a Runge-Kutta fourth order method. The coefficient values are: $A=2$, $B=C=1, \gamma=10, \lambda=0.6, \alpha_{1}=1, d_{1}=0.8957, \alpha_{2}=0.8728, d_{2}=1, K_{1}=2.3831, K_{2}=2.4401$.
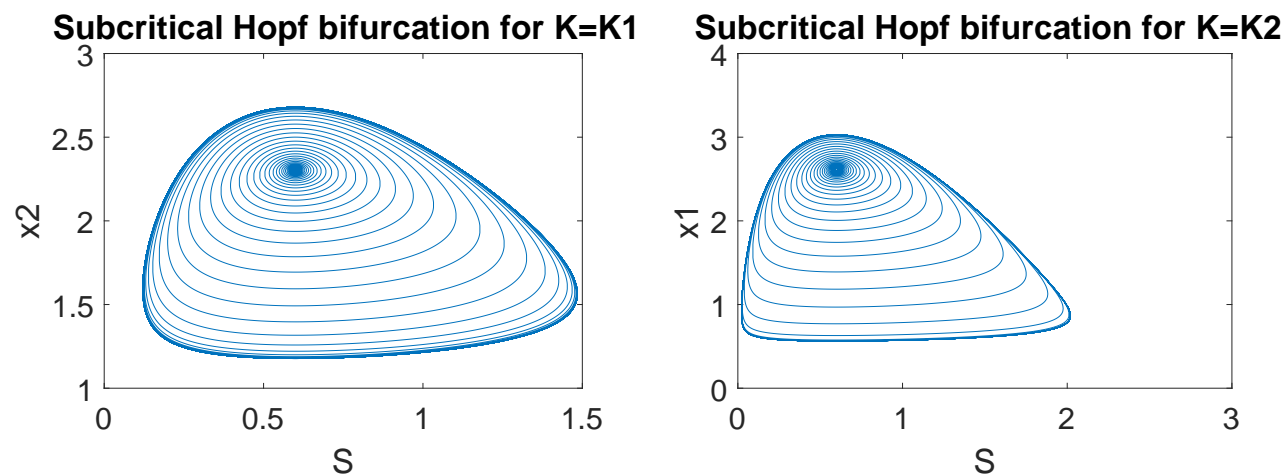

Figure 4. Hopf bifurcation for points $P_{2}\left(K_{1}\right)$ and $P_{1}\left(K_{2}\right)$. Those numerical solutions were generated using a Runge-Kutta fourth order method.

Vol. 35, No. 1, 2017] 
Theorem 5.1. Consider the system:

$$
\begin{aligned}
& \dot{S}=\gamma S\left(1-\frac{3}{\pi} \operatorname{arcsec}\left(\frac{S}{K}+1\right)\right)-x_{i} \frac{A}{B \alpha_{i}+C} \operatorname{arcsec}\left(1+\frac{S}{\alpha_{i}}\right), \\
& \dot{x_{i}}=x_{i} \frac{A}{B \alpha_{i}+C} \operatorname{arcsec}\left(1+\frac{S}{\alpha_{i}}\right)-d_{i} x_{i}
\end{aligned}
$$

with $(i=1,2)$, and assume that $K>\lambda$. Then the steady state points

$$
P_{i}(K)=\left(\lambda, \frac{\gamma \lambda\left(1-\frac{3}{\pi} \operatorname{arcsec}\left(1+\frac{\lambda}{K}\right)\right)}{\frac{A}{B \alpha_{i}+C} \operatorname{arcsec}\left(1+\frac{\lambda}{\alpha_{i}}\right)}\right) \text {, with } i=1,2,
$$

fall in a subcritical hopf bifurcation for $K=K_{3-i}$, i.e., each critical point generates an unstable limit cycle.

Proof. We follow the ideas as in [8]. On linearizing system $(11)$ at $P_{i}(K)$, the eigenvalues turn out to be

$$
\mu_{1,2}(K)=\alpha(K) \pm i \beta(K)
$$

where

$$
\alpha(K)=-\frac{\gamma}{2 p\left(\lambda, \alpha_{i}\right)}\left[-\lambda g_{s}^{\prime}(\lambda, K) p\left(\lambda, \alpha_{i}\right)+g(\lambda, K)\left(\lambda p_{s}^{\prime}\left(\lambda, \alpha_{i}\right)-p\left(\lambda, \alpha_{i}\right)\right)\right]
$$

and

$$
\begin{array}{r} 
\pm i \beta(K)=\frac{1}{2 p\left(\lambda, \alpha_{i}\right)}\left\{\gamma^{2}\left[-\lambda g_{s}^{\prime}(\lambda, K) p\left(\lambda, \alpha_{i}\right)+g(\lambda, K)\left(\lambda p_{s}^{\prime}\left(\lambda, \alpha_{i}\right)-p\left(\lambda, \alpha_{i}\right)\right)\right]^{2}\right. \\
\left.-4 \gamma \lambda g(\lambda, K) p^{2}\left(\lambda, \alpha_{i}\right) p_{s}^{\prime}\left(\lambda, \alpha_{i}\right)\right\}^{\frac{1}{2}} .
\end{array}
$$

Recalling (10), for $i=1,2$,

$$
x_{i}(K)=(-1)^{i} \gamma \frac{-\lambda g_{s}^{\prime}(\lambda, K) p\left(\lambda, \alpha_{3-i}\right)+g(\lambda, K)\left[\lambda p_{s}^{\prime}\left(\lambda, \alpha_{3-i}\right)-p\left(\lambda, \alpha_{3-i}\right)\right]}{p\left(\lambda, \alpha_{2}\right) p_{s}^{\prime}\left(\lambda, \alpha_{1}\right)-p_{s}^{\prime}\left(\lambda, \alpha_{2}\right) p\left(\lambda, \alpha_{1}\right)} .
$$

Provided that our particular model is natural, the denominator is positive, and given that $x_{i}\left(K_{i}\right)=0$ for $i=1,2$, then the numerator of $x_{3-i}\left(K_{3-i}\right)=0$, that is:

$$
\left[-\lambda g_{s}^{\prime}\left(\lambda, K_{3-i}\right) p\left(\lambda, \alpha_{i}\right)+g\left(\lambda, K_{3-i}\right)\left(\lambda p_{s}^{\prime}\left(\lambda, \alpha_{i}\right)-p\left(\lambda, \alpha_{i}\right)\right)\right]=0, \quad i=1,2 .
$$

Therefore,

$$
\beta\left(K_{3-i}\right)=\left\{\gamma \lambda g\left(\lambda, K_{3-i}\right) p_{s}^{\prime}\left(\lambda, \alpha_{i}\right)\right\}^{\frac{1}{2}} .
$$

Now, from (12), we can conclude that, for $i=1,2$,

$$
\gamma \lambda g\left(\lambda, K_{3-i}\right) p_{s}^{\prime}\left(\lambda, \alpha_{i}\right)=p\left(\lambda, \alpha_{i}\right)\left[\lambda g_{s}^{\prime}\left(\lambda, K_{3-i}\right)+g\left(\lambda, K_{3-i}\right)\right] .
$$


So, we have

$$
\beta\left(K_{3-i}\right)=\left\{\gamma p\left(\lambda, \alpha_{i}\right)\left[\lambda g_{s}^{\prime}\left(\lambda, K_{3-i}\right)+g\left(\lambda, K_{3-i}\right)\right]\right\}^{\frac{1}{2}} .
$$

Now, according to Hopf Bifurcation Theorem (see [9]), in order to our system exhibits a bifurcating closed orbit, we need to prove that

$$
\alpha\left(K_{3-i}\right)=0, \quad \beta\left(K_{3-i}\right)>0, \quad \text { and } \quad \alpha_{K}^{\prime}\left(K_{3-i}\right)>0 .
$$

From (12), note that for $i=1,2$,

$\alpha\left(K_{3-i}\right)=-\frac{\gamma}{2 p\left(\lambda, \alpha_{i}\right)}\left[-\lambda g_{s}^{\prime}\left(\lambda, K_{3-i}\right) p\left(\lambda, \alpha_{i}\right)+g\left(\lambda, K_{3-i}\right)\left(\lambda p_{s}^{\prime}\left(\lambda, \alpha_{i}\right)-p\left(\lambda, \alpha_{i}\right)\right)\right]=0$.

It is clear that $\beta\left(K_{3-i}\right)=\left\{\gamma p\left(\lambda, \alpha_{i}\right)\left[\lambda g_{s}^{\prime}\left(\lambda, K_{3-i}\right)+g\left(\lambda, K_{3-i}\right)\right]\right\}^{\frac{1}{2}}>0$. A calculation yields to $\alpha_{K}^{\prime}\left(K_{3-i}\right)=-\frac{\gamma}{2}\left[-\lambda g_{S K}^{\prime \prime}\left(\lambda, K_{3-i}\right)+g_{K}^{\prime}\left(\lambda, K_{3-i}\right)\left(\lambda p_{S}^{\prime}\left(\lambda, \alpha_{i}\right)-p\left(\lambda, \alpha_{i}\right)\right)\right]$. It is easy to note that, for $\lambda<K, g_{K}^{\prime}(\lambda, K)>0$, and from conditions (II) and (VI), we conclude that $\alpha_{K}^{\prime}\left(K_{3-i}\right)>0$. Finally, it is time-consuming to prove that the Hopf bifurcation is subcritical, which is a consequence from the fact that the real part of the Floquet exponent

$$
G(S)=\left(\frac{f^{\prime \prime}(S)}{p_{i}(S) p_{i}^{\prime}(S)}\right)^{\prime} p_{i}^{2}(S) p_{i}^{\prime 2}(S)+\left(\frac{f(S) p_{i}^{\prime}(S)}{p_{i}^{\prime \prime}(S)}\right)^{\prime} p_{i}^{\prime \prime 2}
$$

is positive at $S=\lambda$, where $f(S)=g\left(S, K_{3-i}\right)$ and $P_{i}(S)=p\left(S, \alpha_{i}\right), i=1,2$.

It is important to note that when we take initial conditions outside the cycles, the distance (in a suitable norm) between the respective solution and the limit cycle goes to infinity very fast. From the point of view of competition, this means that in a one-predator one-prey system, both species could or could not survive in the long run, depending on initial conditions. If those conditions (initial quantities of predator and prey) are close to certain numbers (the steady state point), then both species will survive. Nevertheless, if initial quantities of predator and prey are far enough from the steady state point, then predator species or even both species will die out.

\section{Conclusions and discussion}

We have seen that our model for two predators and one prey exhibits the zip bifurcation phenomenon; nevertheless, we have also seen that this model, in general, does not satisfy the so called competitive exclusion principle, according to which "two species of approximately the same food habits are not likely to remain long enough evenly balanced in numbers in the same region. One will crowd out the other" (see [16]).

This principle has been known under many names: Gause's Principle, Gause's Rule, Gause's Law, Gause's Hypothesis, Volterra-Gause Principle, Grinnells Axiom, and Volterra-Lotka Law [17]. From these names, we can notice that the status of the principle is not clear (law, rule, axiom). Moreover, the principle has been highly controversial

Vol. 35, No. 1, 2017] 
not only for the much evidence of coexistence among population species that share a common habitat but also because "empirical studies can not prove and hardly falsify the competitive exclusion principle, it can be verified only theoretically" ([17]).

Our purpose is not to participate in the controversy. Our model is theoretical and clearly only satisfies the competitive exclusion principle when the carrying capacity of the habitat indefinitely grows. In this case, the competitive exclusion principle is satisfied in spite of the excess of food at disposal for the prey species. This is contrary to intuition because one thinks that the more food in one habitat, the better opportunities for everybody to survive. As we said earlier, this situation is known as enrichment paradox, and clearly says that our system could model a very special kind of population species; it may be a special type of bacteria, with neither intuitive nor evident dynamics. The data of real competing population species that behave close to our model may be found in the future by biologists strongly interested on population dynamics.

In memory of Miklos Farkas(1932-2007), our dearest professor.

\section{References}

[1] Angulo F., Olivar G., Osorio G.A., Escobar C.M., Ferreira J.D. and Redondo J.M., "Bifurcations of non-smooth systems", Commun. Nonlinear Sci. Numer. Simul. 17 (2012), No. $12,4683-4689$.

[2] Bocsó A. and Farkas M.,"Political and economic rationality leads to velcro bifurcation", Appl. Math. Comput. 140 (2003), No. 2-3, 381-389.

[3] Butler G.J. and Waltman P., "Bifurcation from a limit cycle in a two predator one prey ecosystem modeled in a chemostat", J. Math. Biol. 12 (1981), No. 3, 295-310.

[4] Butler G.J., Hsu S.B. and Waltman P., "Coexistence of competing predators on a chemostat", J. Math. Biol. 17 (1983), No. 2, 133-151.

[5] Butler G.J., "Competitive predator-prey systems and coexistence", in: Population Biology Proceedings, Edmonton 1982, Lecture Notes in Biomath. 52, Springer-Verlag (1983), 210217.

[6] Escobar-Callejas C.M., Gonzalez-Granada J.R. and Posso-Agudelo A.E., "Atractividad local en la bifurcación zip", Ingeniería y Ciencia 6 (2010), No. 12, 11-41.

[7] Farkas M., "Zip Bifurcation in a competition model", Nonlinear Anal. 8 (1984), No. 11, 1295-1309.

[8] Farkas M., "Competitive exclusion by zip bifurcation", in: Dynamical systems, IIASA Workshop, Sopron, Lecture Notes in Econom. and Math. Systems 287, Springer-Verlag (1987), $165-178$.

[9] Farkas M., Periodic Motions, Springer, New York, 1994.

[10] Farkas M., Dynamical Models in Biology, Academic Press, San Diego, 2001.

[11] Farkas M., Sáez E. and Szántó I., "Velcro bifurcation in competition models with generalized Holling functional response", Miskolc Math. Notes 6 (2005), No. 2, 185-195.

[Revista Integración, temas de matemáticas 
[12] Farkas M. and Ferreira J.D., "Zip bifurcation in a reaction-diffusion system", Differ. Equ. Dyn. Syst. 15 (2007), No. 3-4, 169-183.

[13] Ferreira J.D., "Zip bifurcation in an ample class of competitive systems", Miskolc Math. Notes 8 (2007), No. 2, 147-156.

[14] Ferreira J.D. and De Oliveira L.A.F., "Zip bifurcation in a competitive system with diffusion", Differ. Equ. Dyn. Syst. 17 (2009), No. 1-2, 37-53.

[15] Ferreira J.D., and Sree Hari Rao V., "Unsustainable zip-bifurcation in a predator-prey model involving discrete delay", Proc. Roy. Soc. Edinburgh Sect. A 143 (2013), No. 6, $1209-1236$.

[16] Hardin G., "The competitive exclusion principle", Science 131 (1960), No. 3409, 1292-1297.

[17] Kalmykov L. and Kalmykov V., "Verification and reformulation of the competitive exclusion principle", Chaos Solitons Fractals 56 (2013), 124-131.

[18] May R.M. and McLean A.R., Theoretical ecology. Principles and applications, Oxford University Press, Oxford, 2007.

[19] Sáez E., Stange E. and Szántó I., "Simultaneous zip bifurcation and limit cycles in three dimensional competition models", SIAM J. Appl. Dyn. Syst. 5 (2006), No. 1, 1-11.

[20] Smith H.L. and Waltman P., The theory of the chemostat. Dynamics of microbial competition, Cambridge Studies in Mathematical Biology 13, Cambridge University Press, Cambridge, 1995.

[21] Waltman P., Hsu S.H. and Hubbell S.P., "Theoretical and experimental investigations of microbial competition in continuous cultures. Modelling and differentiel equations in Biology", in Lecture Notes in Pure and Applied Mathematics 58, Dekker (1980), 107-152.

Vol. 35, No. 1, 2017] 